\title{
Effects of Syndiotacticity on the Dynamic and Static Phase Separation Properties of Poly $(N$-isopropylacrylamide) in Aqueous
}

\section{Solution}

\section{Takanori Tada, Tomohiro Hirano, Koichi Ute, Yukiteru Katsumoto, Taka-Aki Asoh, Tatsuya Shoji, Noboru Kitamura, Yasuyuki Tsuboi}

\begin{tabular}{|c|l|}
\hline Citation & The Journal of Physical Chemistry B, 120 (31); 7724-7730 \\
\hline Issue Date & $2016-07-14$ \\
\hline Type & Journal Article \\
\hline Textversion & author \\
\hline $\begin{array}{c}\text { Supporting } \\
\text { Information }\end{array}$ & $\begin{array}{l}\text { The Supporting Information is available free of charge on the ACS Publications } \\
\text { website at https://doi.org/10.1021/acs.jpcb.6b03200. }\end{array}$ \\
\hline Rights & $\begin{array}{l}\text { This document is the Accepted Manuscript version of a Published Work that appeared } \\
\text { in final form in The Journal of Physical Chemistry B, copyright C American Chemical } \\
\text { Society after peer review and technical editing by the publisher. To access the final } \\
\text { edited and published work see https://doi.org/10.1021/acs.jpcb.6b03200 }\end{array}$ \\
\hline DOI & \begin{tabular}{l}
$10.1021 / a c s . j p c b .6 b 03200$ \\
\hline
\end{tabular}
\end{tabular}

Self-Archiving by Author(s)

Placed on: Osaka City University

Takanori Tada, Tomohiro Hirano, Koichi Ute, Yukiteru Katsumoto, Taka-Aki Asoh, Tatsuya Shoji, Noboru Kitamura, and Yasuyuki Tsuboi. (2016). 1.Effects of Syndiotacticity on the Dynamic and Static Phase Separation Properties of Poly(N-isopropylacrylamide) in Aqueous Solution. The Journal of Physical Chemistry B. 120 (31), 7724-7730. 
This document is confidential and is proprietary to the American Chemical Society and its authors. Do not copy or disclose without written permission. If you have received this item in error, notify the sender and delete all copies.

\section{Effects of Syndiotacticity on the Dynamic and Static Phase Separation Properties of Poly( $\mathbf{N}$-Isopropylacrylamide) in Aqueous Solution}

\begin{tabular}{|r|l|}
\hline Journal: & The Journal of Physical Chemistry \\
\hline Manuscript ID & jp-2016-03200a.R2 \\
\hline Manuscript Type: & Article \\
\hline Date Submitted by the Author: & n/a \\
\hline Complete List of Authors: & $\begin{array}{l}\text { Tada, Takanori; Hokkaido Univ., Chemistry, School of Science } \\
\text { Hirano, Tomohiro; Institute of Technology and Science, } \\
\text { Ute, Koichi; Tokushima Daigaku Kogakubu, } \\
\text { Katsumoto, Yukiteru; Fukuoka University } \\
\text { Asoh, Taka-Aki; Osaka City University, } \\
\text { Shoji, Tatsuya; Osaka City University, Graduate School of Science } \\
\text { Kitamura, Noboru; Hokkaido University, Division of Chemistry, Graduate } \\
\text { School of Science } \\
\text { Tsuboi, Yasuyuki; Osaka City Univ., Chemistry, School of Sci. }\end{array}$ \\
\hline
\end{tabular}


Effects of Syndiotacticity on the Dynamic and Static Phase Separation Properties of Poly( $N$-isopropylacrylamide) in Aqueous Solution

Takanori Tada $^{1)}$, Tomohiro Hirano ${ }^{2)}$, Koichi Ute ${ }^{2)}$, Yukiteru Katsumoto ${ }^{3)}$, Taka-Aki Asoh ${ }^{4)}$, Tatsuya Shoji ${ }^{5)}$, Noboru Kitamura ${ }^{1,6)^{*}}$, and Yasuyuki Tsuboi ${ }^{5{ }^{*}}$

${ }^{1}$ Graduate School of Chemical Sciences and Engineering, Hokkaido University, Sapporo 060-0810, Japan

${ }^{2}$ Department of Chemical Science and Technology, Institute of Technology and Science, The University of Tokushima, Minamijosanjima 2-1, Tokushima 770-8506, Japan

${ }^{3}$ Department of Chemistry, Faculty of Science, Fukuoka University,

Jonan-ku, Fukuoka 814-0180, Japan

${ }^{4}$ Advanced Research Institute of Natural Science and Technology,

Osaka City University, 3-3-138 Sugimoto Sumiyoshi-ku, Osaka-shi, 558-8585, Japan

${ }^{5}$ Division of Molecular Material Science, Graduate School of Science,

Osaka City University, 3-3-138, Sugimoto, Sumiyoshi0ku, Osaka 558-8585, Japan

${ }^{6}$ Division of Chemistry, Graduate School of Science, Hokkaido University, Sapporo 060-0810, Japan.

* corresponding author E-mail: kitamura@sci.hokudai.ac.jp (NK), twoboys@sci.osaka-cu.ac.jp (YT) Phone: +81-11-706-2697 (NK), +81-6-6605-2505 (YT) 


\section{Abstract}

The dynamic and static phase separation behavior in aqueous poly( $N$-isopropylacrylamide) (PNIPAM) solutions is highly sensitive to the tacticity of PNIPAM. We investigated the phase separation dynamics of aqueous solutions of PNIPAM with different tacticities (atactic and syndiotactic-rich types), and found that the phase separation dynamics of syndiotactic-rich PNIPAM was much different from that of atactic-type PNIPAM. First, phase separation in syndiotactic-rich PNIPAM was faster. Second, there was a critical point $\left(C_{\mathrm{cp}}\right)$ in the concentration dependence of the phase separation rate: the phase separation accelerated dramatically when the solution concentration was higher than $2.0 \mathrm{wt} \%\left(=C_{\mathrm{cp}}\right)$. Third, syndiotactic-rich PNIPAM required a higher thermal energy for phase separation compared to atactic PNIPAM. Such behavior can be explained on the basis of the high hydrophobicity of syndiotactic-rich PNIPAM in a dehydrated state and a diffusion-controlled aggregation model. The present study shows that precise control of the stereoregularity will open new channels toward the design and development of stimuli-responsive-polymer-based smart materials. 


\section{Introduction}

Poly ( $N$-isopropylacrylamide) (PNIPAM) is a representative thermo-responsive polymer exhibiting coil-to-globule phase transition followed by phase separation in water. ${ }^{1}$ Because of its sharp and reversible transition behavior at approximately $32{ }^{\circ} \mathrm{C}$, this phenomenon has attracted much attention from the viewpoints of fundamentals and applications. $^{2,3}$ On the basis of the experimental evidence in turbidimetry, ${ }^{4,5}$ calorimetry, ${ }^{4,6,7}$ light scattering measurements, ${ }^{4,8}$ infrared spectroscopy, ${ }^{9,10}$ dielectric relaxation measurements ${ }^{11,12}$ and fluorescence spectroscopy, ${ }^{13}$ the following macroscopic phase separation mechanism has been derived. At room temperature, PNIPAM chains homogeneously dissolve in water forming randomly coiled structures upon hydration. Upon heating above a lower critical solution temperature (LCST), cooperative dehydration of the coiled structures due to the thermal energy occurs and they collapse into a globular state. Subsequently, the globular PNIPAM chains aggregate due to hydrophobic interactions resulting in phase separation into PNIPAM-rich domains and water-rich domains. Eventually, the aqueous PNIPAM solution becomes turbid.

Several research groups have suggested that the phase separation behavior is very sensitive to the tacticity of PNIPAM. Ray et al. reported that the LCST of aqueous PNIPAM solutions got less and the transition curves with respect to temperature became broader as the meso-diad content increased (isotactic-rich). ${ }^{14}$ Katsumoto et al. found that the NIPAM dimer (DNIPAM) with the meso configuration ( $m$-NIPAM-d) was more hydrophobic than DNIPAM with the racemo configuration ( $r$-NIPAM-d). ${ }^{15}$ By means of small angle neutron scattering measurements, Nishi et al. revealed that isotactic-rich 
PNIPAM gradually aggregated with increasing temperature, while atactic PNIPAM showed a sharp response at the $\operatorname{LCST}^{16}$ On the other hand, we originally developed a nanosecond pulsed laser temperature jump (T-jump) technique combined with transient photometry and successfully determined the time constants for phase separation for a series of PNIPAMs. ${ }^{17,18}$ We discovered that a slight increase in the isotacticity of the polymer induced a clear acceleration in the phase separation process. ${ }^{19}$ To delve further into this, we employed a single molecule fluorescent imaging technique and fluorescence correlation spectroscopy. Using these techniques, we revealed that isotactic-rich PNIPAM formed a microscopic inter-chain network in aqueous solution even below the LCST. We concluded that such an inter-chain network plays an important role as a precursor for the rapid phase separation in isotactic-rich PNIPAM systems. ${ }^{19}$ These results indicate that the tacticity of PNIPAM is one of the most crucial factors regulating the phase separation characteristics.

In addition, Hirano et al. has reported some interesting behavior for PNIPAM with a high racemo-diad content (syndiotactic-rich): the LCST becomes higher with increasing syndiotacticity. ${ }^{20}$ Moreover, Mori et al. has reported that cooperative dehydration, which is defined as the length of the sequential polymer chains exhibiting simultaneous dissociation of the hydrating water molecules, increases in syndiotactic-rich poly( $N$ - $n$-propylacrylamide) $(\mathrm{PNNPAM}) .{ }^{21}$ Based on these previous studies, it is fruitful to explore the phase separation dynamics in aqueous syndiotactic-rich PNIPAM solutions. In order to throw some light on the phase separation mechanisms and the dynamics of syndiotactic-rich PNIPAM solutions, we conducted laser T-jump experiments and DLS measurements for PNIPAMs with various racemo-diad content. In this work, we demonstrate that the phase separation rate of 
syndiotactic-rich PNIPAM is much faster than that of atactic PNIPAM in the relatively higher concentration (semi-dilute concentration) region. We discuss the phase separation mechanism for syndiotactic-rich PNIPAM in terms of the hydrophobic properties and a diffusion-controlled aggregation model.

\section{Experimental}

\section{Sample preparation and characterization}

$N$-isopropylacrylamide monomer (Wako Pure Chemicals Co., Ltd., 98\%) was repetitively recrystallized from $n$-hexane (Wako Pure Chemicals Co., Ltd., 96\%). 2,2'-azobis(isobutyronitrile) (AIBN) (Wako Pure Chemicals Co., Ltd., > 98\%) was recrystallized from methanol (Wako Pure Chemicals Co., Ltd., > 99.8\%). Tri- $n$-butylborane $\left(n-\mathrm{Bu}_{3} \mathrm{~B}\right)$ and 3-methyl-3-pentanol (3Me3PenOH) supplied from Aldrich Co., Ltd. were used without further purification for polymerization reactions. Atactic PNIPAM was synthesized by free radical polymerization using AIBN as an initiator. ${ }^{18}$ Syndiotactic-rich PNIPAMs were synthesized using $n-\mathrm{Bu}_{3} \mathrm{~B}$ as an initiator in the presence of $3 \mathrm{Me} 3 \mathrm{PenOH}$ at $-60{ }^{\circ} \mathrm{C}$ in the same manner as previously reported. ${ }^{20}$ The number-averaged molecular weight $\left(M_{\mathrm{n}}\right)$ and polydispersity $\left(M_{\mathrm{w}} / M_{\mathrm{n}}\right)$ of the samples were determined using size exclusion chromatography (SEC) (Tosoh Co., Ltd., HLC 8220 instrument) equipped with TSK gels (Tosoh Co., Ltd., SuperHM-H) using $N, N$-dimethylformamide containing $\mathrm{LiBr}$ as an eluent at $40{ }^{\circ} \mathrm{C}$ with a flow rate of 0.35 $\mathrm{mL} \min ^{-1}$. The SEC chromatogram was calibrated with standard polystyrene samples. The tacticity of the PNIPAMs was determined on the basis of the ${ }^{1} \mathrm{H}$ NMR signals of the methylene groups in deuterated dimethyl sulfoxide (DMSO- $\left.d_{6}\right)$ at $150{ }^{\circ} \mathrm{C}$. The details 
for this have been reported elsewhere. ${ }^{20}$

Table 1 summarizes the results of the characterization of the PNIPAMs used in this study. The sample names are abbreviated according to the racemo-diad content. Table 1 also includes cloud point $\left(T_{c}\right)$ for each sample, which was determined by measurements of temperature-dependent optical transmittance. The polydispersity of the polymers used here was somewhat large polydispersity $\left(M_{w} / M_{n} \sim 2\right)$. However, this never affects discussion seriously, because, the tacticity change gave more significant perturbation to phase separation behavior, as realized in the change in $T_{c}$. Moreover, since all the sample solutions examined here clearly showed single-exponential-behaver, we do not consider that the large polydispersity is important.

DSC (differential scanning calorimetry) measurements were made using a micro calorimeter (Mettler-Toledo) with a cell volume of $100 \mu \mathrm{L}$ at a heating rate of 1.0 ${ }^{\circ} \mathrm{C} \min ^{-1}$ in the range of $25-40{ }^{\circ} \mathrm{C}$ at a raised pressure $\left(>1.1 \times 10^{5} \mathrm{~Pa}\right)$.

DLS (dynamic light scattering) measurements were carried out using a model FDLS-300 (Otsuka Electronics) at $25^{\circ} \mathrm{C}$. A $100 \mathrm{~mW}$ laser $(\lambda=532 \mathrm{~nm})$ was used for the incident beam, and the scattering angle was $90^{\circ}$. We investigated the concentration dependence of the hydrodynamic radius $\left(R_{\mathrm{h}}\right)$ of the polymer in aqueous solutions with various concentrations from 1.0 to $7.0 \mathrm{wt} \%$ (from dilute concentration to semi-dilute concentration).

\section{Laser T-jump photometry}

Two types of laser T-jump technique were employed ${ }^{17}$; (i) a direct heating method where water was directly heated by an infrared laser light pulse and (ii) a dye-sensitized heating method where a dye, used as a molecular heater, was excited by a 
visible laser light pulse. In the former method, (i), a nanosecond $1200 \mathrm{~nm}$ laser pulse was obtained by focusing a $1064 \mathrm{~nm}$ laser light pulse from a $\mathrm{Nd}^{3+}$ : YAG laser (Spectra Physics, PRO-250-10, fwhm 10 ns) into a Raman shifter (Solar Laser, LZ221) where a single $\mathrm{Ba}\left(\mathrm{NO}_{3}\right)_{2}$ crystal was placed in an optical cavity. This was used as the heating light pulse for the T-jump. The heating light pulse was focused onto a sample cell that was maintained at a temperature marginally lower $(0.20 \mathrm{~K})$ than the LCST resulting in phase separation. Namely, the base temperature was set to $0.2 \mathrm{~K}$ lower than cloud point (shown in Table 1) for each sample solution. In this system, a representative temperature rise $(\Delta T)$ is $0.35 \mathrm{~K}$ at a laser fluence $(\lambda=1200 \mathrm{~nm})$ of $0.10 \mathrm{~J} \mathrm{~cm}^{-2}$. A continuous-wave DPSS laser beam (probe light, GC Photonics, GLML-30, $\lambda=532 \mathrm{~nm}$ ) aligned coaxially with the heating light pulse was introduced into the sample cell. Temporal changes in the intensity of the transmitted light were measured with a fast photodiode (THORLABS, DET 210) and a digital oscilloscope (Lecroy, wave Runner 104MXi, $1 \mathrm{GHz})$.

In method (ii), it was possible to control $\Delta T$ by adjusting the concentration of the dye. A small amount of Crystal Violet (CV) (Aldrich Co., Ltd., $0.50 \mathrm{mM}$ ) was added to the sample solution. A $532 \mathrm{~nm}$ laser pulse from the $\mathrm{Nd}^{3+}$ : YAG laser (same as in the method (i)) was used as the heating light pulse. $\Delta T$ was $1.1 \mathrm{~K}$ at a fluence of $0.48 \mathrm{~J} \cdot \mathrm{cm}^{-2}$. The dye molecules absorbed the $532 \mathrm{~nm}$ laser pulse and acted as a molecular heater, converting light energy into heat with high efficiency $(\sim 100 \%)$ through rapid internal conversion coupled with cyclic repetitive absorption. $^{22}$ A continuous-wave semiconductor laser beam $(\lambda=690 \mathrm{~nm})$ (NEOARK, LDP-6935M) was used for the probe light. These two methods gave the same results in terms of the phase separation dynamics. That is, the CV hardly affected the phase separation dynamics, as already 
reported in our previous work. ${ }^{17}$

\section{Results and Discussion \\ Sensitivity of the phase separation to the laser T-jump}

Figure 1 shows a representative time profile of the optical transmittance $(T(t))$ obtained by method (i) for syndiotactic-rich PNIPAM ( $r-68$, red line) at a concentration of $5.0 \mathrm{wt} \%$. Also, a profile for an atactic PNIPAM ( $r-54$, black line) solution $(5.0 \mathrm{wt} \%)$ is shown in the figure as a reference. Since these two polymers have similar molecular weight, we can safely compare them taking only the effect of tacticity into account. For $r-54$, as can be clearly seen in the figure, $T(t)$ begins to decrease immediately after the T-jump, and reaches a quasi-equilibrium state after $200 \mathrm{~ms}$ where the transmittance approaches a constant value. The decay of $T(t)$ is due to an increase in the turbidity of the solution caused by phase separation. The $T(t)$ curve for $r-54$ can be well fitted with a single exponential function, $T(t)=A \exp \left(-t / \tau_{\mathrm{ps}}\right)+B$ (see the white dotted line in the figure), providing us with a precise value of the phase separation time constant $\left(\tau_{\mathrm{ps}}\right)$; this was evaluated to be $77 \mathrm{~ms}$. Generally, according to our past studies, phase separation in PNIPAM systems takes place on a time scale of $40 \sim 200 \mathrm{~ms} .{ }^{18}$ By contrast, for $r-68$, no sign of transmittance decay was observed after the T-jump, although the fluence was the same as in the $r$-54 measurement. Note that the base temperature was $0.2 \mathrm{~K}$ lower that $T_{c}$ both for $\mathrm{r}-54$ and $\mathrm{r}-68$ solutions. This result strongly implies that phase separation in the $r$ - 68 solution requires a higher energy than that in the $r-54$ solution. Similar behavior 
to this was also observed for the other syndiotactic-rich PNIPAMs, $r-67$ and $r-66$.

Since with using method (i) phase separation in the syndiotactic-rich PNIPAMs was not induced, we employed method (ii) and measured the lowest value of the fluence of the heating pulse required for phase separation. The temperature rise is given by,

$$
\Delta T=(I / C L)\left(1-e^{-2.303 \alpha L}\right)
$$

where $I$ is the incident light intensity, $C$ is the heat capacity of the medium $\left(\mathrm{J} \cdot \mathrm{cm}^{-3} \cdot \mathrm{deg}^{-1}\right), L$ is the optical path length, and $\alpha$ is the absorption coefficient $\left(\mathrm{cm}^{-1}\right)$. Here, $C$ and $L$ are the same $\left(c=4.2 \mathrm{~J} \cdot \mathrm{cm}^{-3} \cdot \mathrm{deg}^{-1}, L=0.10 \mathrm{~cm}\right)$ for both methods (i) and (ii). The values of $\alpha$ for methods (i) and (ii) are 1.1 and 104, respectively ${ }^{22}$. Therefore, according to eq. (1), method (ii) induces 5 times higher $\Delta T$ than method (i) for the same value of $I_{\mathrm{IR}}$. Indeed, using method (ii) phase separation of syndiotactic-rich PNIPAMs can be induced.

Figure 2 shows the time profiles of $T(t)$ for (a) $r-54$ and (b) $r-68$ obtained with method (ii) with various heating pulse fluence ranging from 0.10 to $0.39 \mathrm{~J} \mathrm{~cm}^{-2}$. Due to the high absorbance of the $\mathrm{CV}$, method (ii) induces phase separation even in the syndiotactic-rich PNIPAM solutions. Each decay of $T(t)$ can be well fitted with a single exponential function. Therefore, we can precisely determine $\tau_{\mathrm{ps}}$ using method (ii). According to Figure 2, the lowest energy to phase separation for $r-68$ and $r-54$ were $0.16 \mathrm{~J} \cdot \mathrm{cm}^{-2}$ and $0.10 \mathrm{~J} \cdot \mathrm{cm}^{-2}$, respectively. On the basis of these results, it was concluded that syndiotactic-rich PNIPAM solutions require higher energy for phase separation compared with atactic PNIPAM solutions. 
Phase separation rate

Using method (ii), the phase separation time constants $\left(\tau_{\mathrm{ps}}\right)$ were systematically determined for both atactic and syndiotactic-rich PNIPAMs by varying the solution concentration $(0.50 \sim 7.0 \mathrm{wt} \%)$. As a reference, a representative decay of $T(t)$ for $r-54$ is shown in Figure 3a. The decay of $T(t)$ becomes gradually faster with increasing polymer concentration. On the other hand, $r-68$ shows the characteristic concentration dependence as seen in Figure 3b. In the figure, the decay of $T(t)$ clearly becomes faster with increasing concentration. In Figure $4 \mathrm{a}, \tau_{\mathrm{ps}}$ is plotted against polymer concentration for atactic PNIPAM $(r-54)$ and syndiotactic-rich PNIPAM $(r-68)$. It is easily seen that these two samples exhibit different dynamic behavior. In the lower concentration region (dilute solution, $0.50 \sim 1.5 \mathrm{wt} \%$ ), the time constants of $r-54$ and $r-68$ are close to each other $\left(\tau_{\mathrm{ps}}=150 \sim 200 \mathrm{~ms}\right)$. In this region, phase separation in both $r-54$ and $r-68$ gradually accelerate with increasing concentration. By contrast, at $2.0 \mathrm{wt} \%$ ( $C_{\mathrm{cp}}$ : critical concentration), there is an abrupt change in the phase separation in $r-68$ which becomes much faster $\left(\tau_{\mathrm{ps}}=10 \mathrm{~ms}\right)$, while that in $r-54$ still accelerates gradually $\left(\tau_{\mathrm{ps}}=100 \mathrm{~ms}\right)$. This abrupt acceleration was also seen in the decay of $T(t)$ for $r$-68: there is an abrupt increase in the decay of $T(t)$ when the concentration rises above $2.0 \mathrm{wt} \%$. It should be pointed out here that $\tau_{\mathrm{ps}}$ of $r-68$ became constant in the semi-dilute solutions (concentrations $\geq C_{\mathrm{cp}}$ ). Similar behavior with respect to concentration dependence was also observed for the other syndiotactic-rich PNIPAMs, $r-66$ and $r-67$, as shown in Figures $4 \mathrm{~b}, 4 \mathrm{c}$ and S2. What is important here is that the $C_{\mathrm{cp}}$ at which the phase separation accelerates is common to all the syndiotactic-rich PNIPAMs examined here. 
Quantitative analysis

$$
\left\langle M_{\text {agg }}\right\rangle=M_{w}\left(1+\frac{4}{3} N_{A} \frac{k T}{\eta} \frac{C}{M_{w}} t\right)
$$

where $N_{A}$ is Avogadro's number, $k$ is the Boltzmann constant, $T$ is temperature, $\eta$ is the viscosity of the sample solution, and $C$ is the concentration of the aqueous polymer solution. For instance, this equation can be applied to the results for the $r$ - 54 and $r$ - 68 solutions $(1.0 \mathrm{wt} \%)$, where the values of $\eta$ measured using a viscometer (Brookfield, cone plate version) are 1.1 and $1.2 \mathrm{mPa} \cdot \mathrm{s}$, respectively. The polymer weight in a single polymer-rich domain (assuming a droplet), $M_{\text {agg }}$, is given by eq. (3),

$$
M_{\text {agg }}=\frac{4}{3} \pi r^{3} \times 10^{-12} \times C_{\text {globule }}
$$

where $r$ is the radius of the droplet and $C_{\text {globule }}$ is the polymer concentration (wt $\left.\%\right)$ in the domain. Following a laser light scattering study, Wu et al. predicted the polymer density of a PNIPAM globule to be $0.20 \mathrm{~g} \cdot \mathrm{mL}^{-1}$, which corresponds to $C_{\text {globule }}=20 \mathrm{wt} \%{ }^{8}$

The growth time is then given by eq. (4), 


$$
t=\frac{3}{4}\left(N_{g l o} \times M_{w}-M_{w}\right) \frac{\eta}{N_{A} k T c}
$$

where $N_{g l o}$ is the number of polymer chains in a single polymer-rich domain. Based on these relationships, the average number of polymer chains in a single polymer-rich micro-domain $\left(N_{g l o}=<M_{a g g}>/ M_{\mathrm{w}}\right)$ in the $r$-54 and $r$ - 68 solutions is estimated to be 2000 and 1900, respectively. Then, using eq. (3), the growth times of polymer-rich microdomains in the $r-54$ and $r$ - 68 solutions at 1.0 and $5.0 \mathrm{wt} \%$ are estimated to be $\approx 15$ $(1.0 \mathrm{wt} \%)$ and $3.0 \mathrm{~ms}(5.0 \mathrm{wt} \%)$, respectively. These calculated values for $r-54$ are somewhat smaller than the $\tau_{\mathrm{ps}}$ values obtained from experiments $\left(\tau_{\mathrm{ps}}=70 \sim 200 \mathrm{~ms}\right)$. This suggests that the aggregation of globules takes place through a number of collisions between the globules; the aggregation is a reaction-controlled process. This model is applicable to an $r-68$ solution at $1.0 \mathrm{wt} \%$. On the other hand, in case of an $r-68$ solution at $5.0 \mathrm{wt} \%$, the $\tau_{\mathrm{ps}}$ value is $10 \mathrm{~ms}$, which is very close to the calculated value (3.0 ms). This means that every collision between the globules of $r-68$ at $5.0 \mathrm{wt} \%$ results in aggregation; aggregation is thus very efficient.

DSC results

The degree of cooperative dehydration, which is defined as the length of the sequential polymer chains undergoing simultaneous dissociation from the hydrating water molecules, is evaluated on the basis of the heat capacity changes upon phase transition/separation. ${ }^{6,21}$ Figure 5 shows the heat capacity changes during the phase 
transition/separation of aqueous $r-54$ and $r-68$ solutions $(1.0 \mathrm{wt} \%)$ measured at a heating rate of $1.0{ }^{\circ} \mathrm{C} / \mathrm{min}$. With increasing $r$-content, the endothermic peak resulting from dehydration of the polymer chains becomes much sharper and shifts to a higher temperature region. The thermodynamic parameters determined by such DSC measurements are summarized in Table 2. The number of cooperative units $n_{\text {coop }}$, which is defined by the number of monomer units undergoing cooperative dissociation of the polymer chains from the hydrating water molecules, can be calculated from the ratio of the van't Hoff enthalpy $\left(\Delta H_{\mathrm{v}}\right)$ to the calorimetric enthalpy $\left(\Delta H_{\mathrm{c}}\right){ }^{6,21}$ As can clearly be seen in Table 2, the $n_{\text {coop }}$ value increases with increasing $r$-content. The results indicate that the degree of cooperative dehydration increases with increasing $r$-content.

\section{Cloud points and hysteresis}

Figure 6 shows the transmittance curves of aqueous (a) $r-54$ and (b) $r-68$ solutions as a function of temperature. The red and blue curves show the transmittance changes upon heating and cooling, respectively. Upon heating, the LCST increases with increasing $r$-content in the polymer. Furthermore, with increasing $r$-content, the hysteresis between the heating and cooling processes becomes larger: the differences between the transition temperatures in the heating and cooling processes in $r-54$ and $r-68$ are 1.0 and $3.5^{\circ} \mathrm{C}$, respectively. These tendencies are in good agreement with the results reported previously by Hirano et al. $^{25}$ As Hirano et al. and Zhang et al. reported, ${ }^{26,27}$ the hysteresis originates from the formation of intra- or inter-chain hydrogen bonding in a globular state above the LCST. Thus, the large temperature hysteresis in the aqueous syndiotactic-rich PNIPAM solution implies that the polymer 
forms intra-chain hydrogen bonding in the dehydrated state.

\section{DLS results}

To investigate the polymer structures in aqueous solutions, we measured the hydrodynamic radius $\left(R_{\mathrm{h}}\right)$ of each syndiotactic-rich PNIPAM at various concentrations ranging from $0.50 \mathrm{wt} \%$ to $7.0 \mathrm{wt} \%$ using dynamic light scattering (DLS). Figure 7 shows the concentration dependence of the $R_{\mathrm{h}}$ distribution for $r-68$. In the dilute region $(0.50 \mathrm{wt} \% \sim 1.5 \mathrm{wt} \%)$, a distribution with a single peak was observed, indicating no aggregation of polymer chains in the solution. In contrast, above $1.5 \mathrm{wt} \%(2.0,5.0,7.0$ wt $\%$ ), two peaks were detected. This implies the formation of inter-chain interactions such as aggregation. The average size (diameter) of the aggregate in the $r$ - 68 solution $(2.0 \mathrm{wt} \%)$ was $140 \mathrm{~nm}$. Similar behavior was observed for the other syndiotactic-rich PNIPAMs, $r-67$ and $r$-66 as shown in Figure S3. Thus, we discovered a common critical concentration $(2.0 \mathrm{wt} \%)$ for both the dynamic and static properties. At the critical concentration, these properties clearly change as described above. Clearly, such polymer chain aggregation is the origin of the sudden acceleration of phase separation in syndiotactic-rich PNIPAMs.

It should be noted that, to estimate the radius of gyration, DLS should be measured for dilute solution where overlap of polymer chains is negligible. We carried out it to investigate aspects of polymer aggregation. Füllbrandt et al. investigated PNIPAM phase transition by means dielectric spectroscopy. ${ }^{28}$ They revealed that $\Delta \mathrm{LCST}$ (hysteresis difference in LCST; $\triangle \mathrm{LCST}=\mathrm{LCST}_{\text {heating }}$ and $\mathrm{LCST}_{\text {cooling }}$ ) clearly depended upon polymer concentration, based on which they implied polymer 
aggregation at higher concentrations. This is consistent with our model.

\section{Mechanism}

Here, we discuss the origins of the higher energy required for phase separation and of the acceleration of phase separation at higher concentrations (semi-dilute solutions) for syndiotactic-rich PNIPAM. We propose a tentative mechanism for the phase separation process as shown in Figure 8. In a simple framework, phase transition is an equilibrium process and it never needs activation energy. However, the process investigated here consists of complicated multiple processes (dehydration, coil-grobule phase transition, diffusion, association, and so on) and one of such process, dehydration, possibly requires an additional energy as noted below.

The higher energy can be explained in terms of the stability of the NIPAM-dimer in aqueous solution. Katsumoto et al. calculated the stability using molecular dynamics calculations and concluded that the racemo-NIPAM-dimer ( $r$-NIPAM-d) was more stable than the meso-NIPAM-dimer ( $m$-NIPAM-d) because of the balance between the hydration free energy and the conformational entropy. ${ }^{17}$ Thus, syndiotactic-rich PNIPAM requires relatively higher energy for dehydration followed by phase separation than atactic PNIPAM.

Next, we discuss the origin of the acceleration of the phase separation for syndiotactic-rich PNIPAM in the semi-dilute solutions from the viewpoint of the hydrophobicity in a dehydrated state. As already pointed out, syndiotactic-rich PNIPAM undergoes highly cooperative dehydration. Furthermore, as seen in the results of 
transmittance measurements, the formation of intra-chain hydrogen bonds proceeds between adjacent monomer units in the dehydrated state in a syndiotactic-rich PNIPAM solution (Figure 8). Mori et al. also reported the formation of intra-chain hydrogen bonding in the dehydrated state in an aqueous syndiotactic-rich poly( $N$ - $n$-propylacrylamide) (PNNPAM) solution as revealed by FT-IR spectroscopy of the polymer. ${ }^{21}$ This cooperative formation of intra-chain hydrogen bonding presumably makes the dehydrated polymer globules more hydrophobic. Such hydrophobic globules of syndiotactic-rich PNIPAM should have a more compact structure compared to atactic PNIPAM, leading to faster diffusion toward aggregation. As a result, aggregation (phase separation) of the syndiotactic-rich polymer becomes faster compared to that of atactic polymer.

Finally, we discuss the mechanism for the specific concentration dependence of the phase separation rate for syndiotactic-rich PNIPAM. In the semi-dilute solutions (concentrations $\geq 2.0 \mathrm{wt} \%$ ), the polymer chains form aggregates even at room temperature (before phase separation). In this situation, phase separation occurs through two processes; i) shrinkage of the aggregates formed before phase separation, and ii) diffusion and aggregation of single globules (normal phase separation). Although there are two phase separation processes in the semi-dilute solutions, $T(t)$ can be fitted by a single exponential function. In the present study, the phase separation dynamics are characterized by optical transmittance (i.e. Rayleigh light scattering). It is well known that the Rayleigh scattering intensity is proportional to the third power of the diameter of the particles. Therefore, in the semi-dilute solutions, the signal for phase separation mainly arises from shrinkage of the aggregates. The shrinkage is much faster than normal phase separation due to the high hydrophobicity. This process is independent of 
concentration because the polymer chains in the solution need not diffuse for phase separation.

We should recognize that the picture described above is a simplified model. Relationships between molecular interactions (hydrogen bonding, hydrophobic interaction) and macroscopic behaviors still remain unclear under such complex phase separation. Actually, as Philipp revealed for dilute and semi-dilute aqueous PNIPAM solutions, the specific refractivity is much sensitive to the changes in molecular interactions and in structure accompanying phase separation. ${ }^{29}$ This finding strongly suggests that the real picture of phase separation is complicated and should be elucidated taking into account such effects. This will be a challenging task in the next stage of investigation.

\section{Conclusion}

In the present study, we investigated the phase separation dynamics of syndiotactic-rich PNIPAM and compared it with that of atactic PNIPAM. Based on a T-jump study, optical transmittance, and DSC and DLS measurements, the phase separation characteristics of syndiotactic-rich PNIPAM are summarized as follows:

(i) Higher energy is required for phase separation compared to that of atactic PNIPAM.

(ii) The degree of cooperativity in the dehydration process increases, resulting in high hydrophobicity.

(iii) Phase separation become much fast in the semi-dilute solutions 
(concentrations $\geq 2.0 \mathrm{wt} \%$ ).

(iv) Acceleration of the phase separation is induced by aggregation of the polymer chains.

These findings offer a new approach to the design and development of stimuli-responsive-polymer-based smart materials.

\section{Associated Content}

Supporting Information Phase separation time constant as a function of laser fluence, time profiles of the optical transmittance, phase separation time constant as a function of concentration and representative results on the concentration dependence of the $R_{\mathrm{h}}$ distribution for aqueous solutions of $r-67$ and $r$-66. This material is available free of charge via the Internet at http://pubs.acs.org.

\section{Acknowledgement}

This work was supported by JSPS KAKENHI Grant Numbers JP26288011, JP14J02533, and partly JP16H06507 in Scientific Research on Innovative Areas "Nano-Material Optical-Manipulation”. 


\section{References}

(1) Heskins, M.; Guillet, J. E. Solution Properties of Poly( $N$-isopropylacrylamide). $J$. Macromol. Sci. Part A - Chem. 1968, 2, 1441-1455.

(2) Schild, H. G. Poly( $N$-isopropylacrylamide): Experiment, Theory and Application. Prog. Polym. Sci. 1992, 17, 163-249.

(3) Gil, E.; Hudson, S. Stimuli-Reponsive Polymers and Their Bioconjugates. Prog. Polym. Sci. 2004, 29, 1173-1222.

(4) Kubota, K.; Fujishige, S.; Ando, I. Solution Properties of poly( $N$-isopropylacrylamide) in Water.pdf. Polym. J. 1990, 22, 15-20.

(5) Tong, Z.; Zeng, F.; Zheng, X.; Sato, T. Inverse Molecular Weight Dependence of Cloud Points for Aqueous Poly( $N$-isopropylacrylamide) Solutions. Macromolecules 1999, 32, 4488-4490.

(6) Tiktopulo, E. I.; Bychkova, V. E.; Ricka, J.; Ptitsyn, O. B. Cooperativity of the Coil-Globule Transition in a Homopolymer: Microcalorimetric Study of Poly( $N$-isopropylacrylamide). Macromolecules 1994, 27, 2879-2882.

(7) Durme, K. Van; Assche, G. Van; Mele, B. Van. Kinetics of Demixing and Remixing in Poly( $N$-isopropylacrylamide )/ Water Studied by Modulated Temperature DSC. Macromolecules 2004, 37, 9596-9605.

(8) Wu, C.; Zhou, S. Laser Light Scattering Study of the Phase Transition of Poly( $N$-isopropylacrylamide) in Water. 1. Single Chain. Macromolecules 1995, 28, 8381-8387.

(9) Maeda, Y.; Higuchi, T.; Ikeda, I. Change in Hydration State during the Coil-Globule Transition of Aqueous Solutions of Poly( $N$-isopropylacrylamide) as Evidenced by FTIR Spectroscopy. Langmuir 2000, 16, 7503-7509.

(10) Sun, B.; Lin, Y.; Wu, P.; Siesler, H. W. A FTIR and 2D-IR Spectroscopic Study on the Microdynamics Phase Separation Mechanism of the Poly ( $N$-isopropylacrylamide) Aqueous Solution. Macromolecules 2008, 41, 15121520.

(11) Ono, Y.; Shikata, T. Hydration and Dynamic Behavior of Poly( $N$-isopropylacrylamide)s in Aqueous Solution: A Sharp Phase Transition at the Lower Critical Solution Temperature. J. Am. Chem. Soc. 2006, 128, 1003010031. 
(12) Füllbrandt, M.; Ermilova, E.; Asadujjaman, A.; Hölzel, R.; Bier, F. F.; von Klitzing, R.; Schönhals, A. Dynamics of Linear Poly( $N$-isopropylacrylamide) in Water around the Phase Transition Investigated by Dielectric Relaxation Spectroscopy. J. Phys. Chem. B 2014, 118, 3750-3759.

(13) Press, M. M. I.; Winnik, F. M. Fluorescence Studies of Aqueous Solutions of Poly( $N$-isopropylacrylamide ) below and above Their LCST. Macromolecules 1990, 23, 233-242.

(14) Ray, B.; Okamoto, Y.; Kamigaito, M.; Sawamoto, M.; Seno, K.; Kanaoka, S.; Aoshima, S. Effect of Tacticity of Poly( $N$-isopropylacrylamide) on the Phase Separation Temperature of Its Aqueous Solutions. Polym. J. 2005, 37, 234-237.

(15) Katsumoto, Y.; Kubosaki, N.; Miyata, T. Molecular Approach to Understand the Tacticity Effects on the Hydrophilicity of $\operatorname{Poly}(N$-isopropylacrylamide): Solubility of Dimer Model Compounds in Water. J. Phys. Chem. B 2010, 114, 13312-13318.

(16) Nishi, K.; Hiroi, T.; Hashimoto, K.; Fujii, K.; Han, Y.; Kim, T.-H.; Katsumoto, Y.; Shibayama, M. SANS and DLS Study of Tacticity Ffects on Hydrophobicity and Phase Separation of Poly( $N$-isopropylacrylamide). Macromolecules 2013, 46, 6225-6232.

(17) Tsuboi, Y.; Yoshida, Y.; Okada, K.; Kitamura, N. Phase Separation Dynamics of Aqueous Solutions of Thermoresponsive Polymers Studied by a Laser T-Jump Technique. J. Phys. Chem. B 2008, 112, 2562-2565.

(18) Tsuboi, Y.; Tada, T.; Shoji, T.; Kitamura, N. Phase-Separation Dynamics of Aqueous Poly( $N$-isopropylacrylamide) Solutions: Characteristic Behavior of the Molecular Weight and Concentration Dependences. Macromol. Chem. Phys. 2012, 213, 1879-1884.

(19) Tada, T.; Katsumoto, Y.; Goossens, K.; Uji-i, H.; Hofkens, J.; Shoji, T.; Kitamura, N.; Tsuboi, Y. Accelerating the Phase Separation in Aqueous Poly( $N$-isopropylacrylamide) Solutions by Slight Modification of the Polymer Stereoregularity: A Single Molecule Fluorescence Study. J. Phys. Chem. C 2013, $117,10818-10824$.

(20) Hirano, T.; Okumura, Y.; Kitajima, H.; Seno, M.; Sato, T. Dual Roles of Alkyl Alcohols as Syndiotactic-Specificity.pdf. J. Polym. Sci. Part A Polym. Chem. 2006, 44, 4450-4460.

(21) Mori, T.; Hirano, T.; Maruyama, A.; Katayama, Y.; Niidome, T.; Bando, Y.; Ute, 
K.; Takaku, S.; Maeda, Y. Syndiotactic $\operatorname{Poly}(N-n$-Propylacrylamide) Shows Highly Cooperative Phase Transition. Langmuir 2009, 25, 48-50.

(22) Madge, D.; Windsor, M. W. Picosecond Internal Conversion in Crystal Violet. Chem. Phys. Lett. 1974, 24, 144-148.

(23) Chu, B.; Ying, Q.; Grosberg, A. Y. Two-Stage Kinetics of Single-Chain Collapse. Polystyrene in Cyclohexane. Macromolecules 1995, 28, 180-189.

(24) Nakamura, Y.; Sasaki, N.; Nakata, M. Chain Aggregation Process of Poly(methyl methacrylate) in the Mixed Solvent tert-Butyl Alcohol + Water. Macromolecules 2002, 35, 1365-1372.

(25) Hirano, T.; Miki, H.; Seno, M.; Sato, T. Effect of Polymerization Conditions on the Syndiotactic-Specificity in Radical Polymerization of $N$-isopropylacrylamide and Fractionation of the Obtained Polymer according to the Stereoregularity. Polymer. 2005, 46, 5501-5505.

(26) Hirano, T.; Yamamoto, H.; Ute, K. Effects of Chemical Composition and Stereoregularity on Phase-Transition Behaviors of Aqueous Solutions of Copolymers Composed of $N$-isopropylacrylamide and $N$-n-propylacrylamide. Polymer. 2011, 52, 5277-5281.

(27) Lu, Y.; Zhou, K.; Ding, Y.; Zhang, G.; Wu, C. Origin of Hysteresis Observed in Association and Dissociation of Polymer Chains in Water. Phys. Chem. Chem. Phys. 2010, 12, 3188-3194.

(28) Füllbrandt, M.; von Klitzing, R.; Schönhals, A. Probing the Phase Transition of Aqueous Solutions of Linear Low Molecular Weight Poly( $N$-isopropylacrylamide) by Dielectric Spectroscopy. Soft Matter 2012, 8 , 12116-12123.

(29) Philipp, M.; Aleksandrova, R.; Müller, U.; Ostermeyer, M.; Sanctuary, R.; Müller-Buschbaum, P.; Krüger, J. K. Molecular versus Macroscopic Perspective on the Demixing Transition of Aqueous PNIPAM Solutions by Studying the Dual Character of the Refractive Index. Soft Matter 2014, 10, 7297-7305. 
Table 1. $\quad$ Fundamental properties of PNIPAMs.

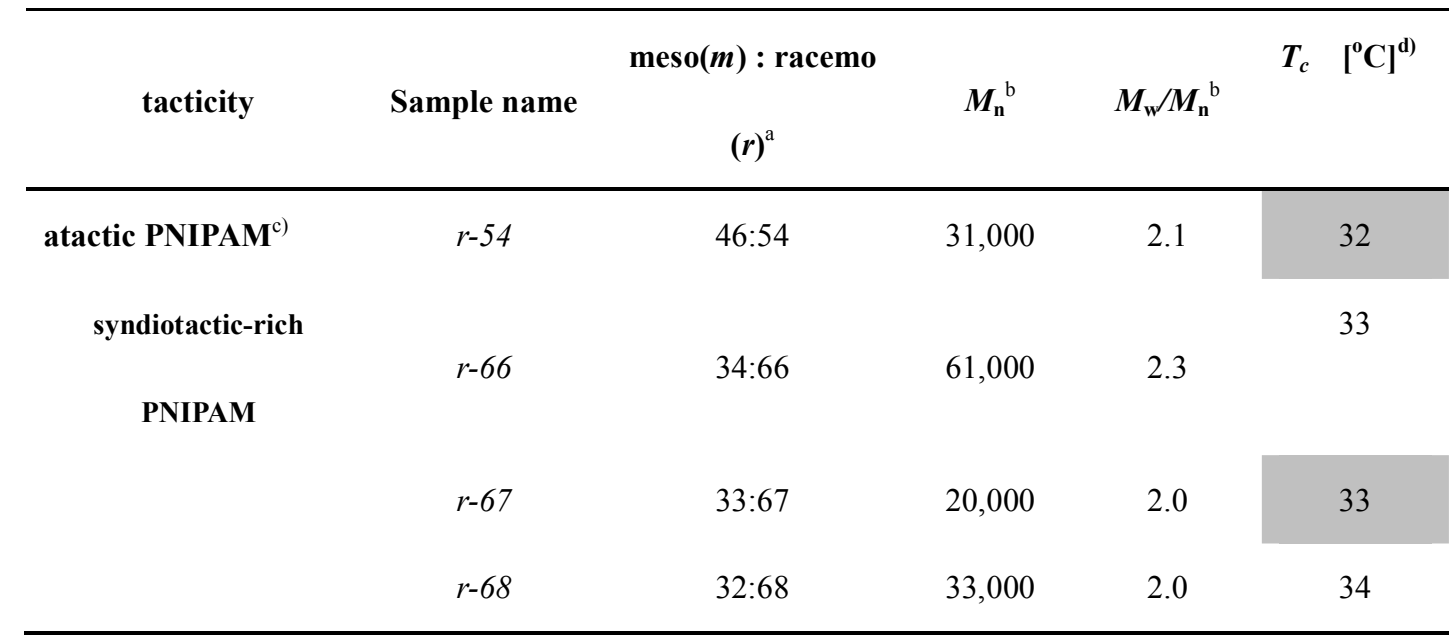

(a) Determined by ${ }^{1} \mathrm{H}$ NMR signals. Error of the value is less than $1.0 \%$

(b) Determined by SEC (polystyrene standards).

(c) $r$-54 PNIPAM is not perfectly atactic. However, it is the closest to atactic PNIPAM among the PNIPAMs in the table.

d) Error of the value is less than $1.0 \%$ 


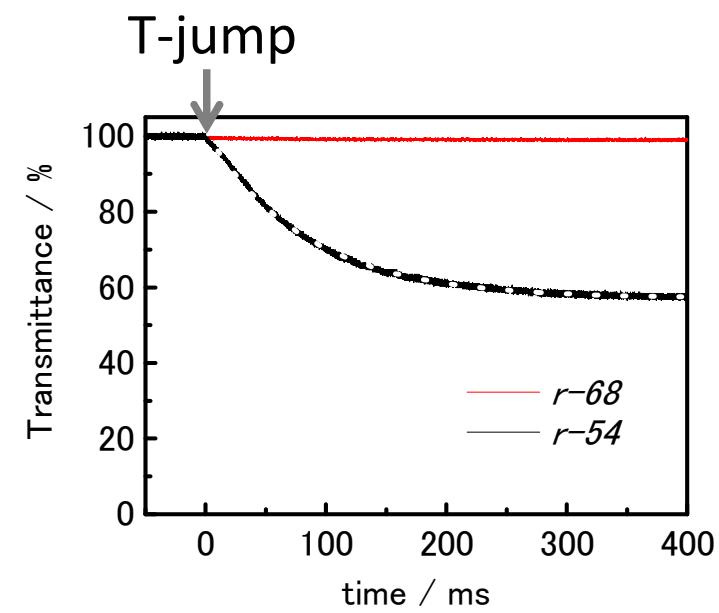

Figure 1. Time profiles of the optical transmittance of $r$-54 (black line) and $r-68$ (red line). The concentration of the solutions is $5.0 \mathrm{wt} \%$. The white dotted line on the r-54 curve shows the results fitted by a single exponential function $\left(T(t)=A \exp \left(-t / \tau_{\mathrm{ps}}\right)\right.$ $+B$ ). The base temperature (temperature before T-jump) was $31.8{ }^{\circ} \mathrm{C}$ for $r$-54 and 33.8 ${ }^{\circ} \mathrm{C}$ for $r-68$. 
(a) $r-54$

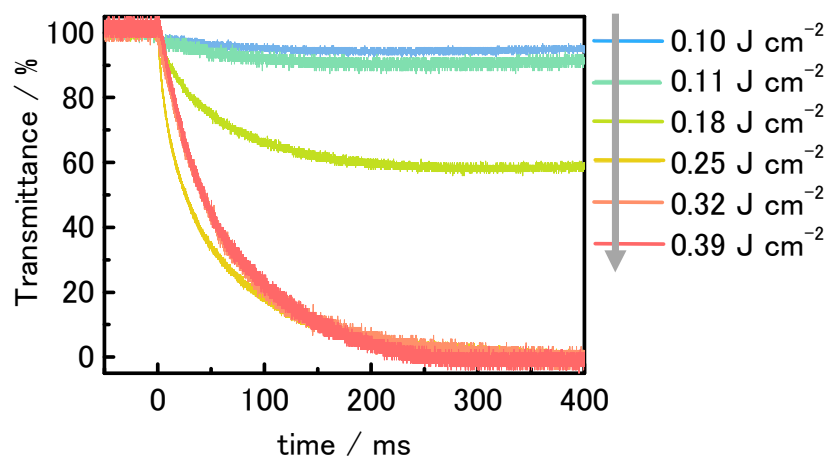

(b) $r-68$

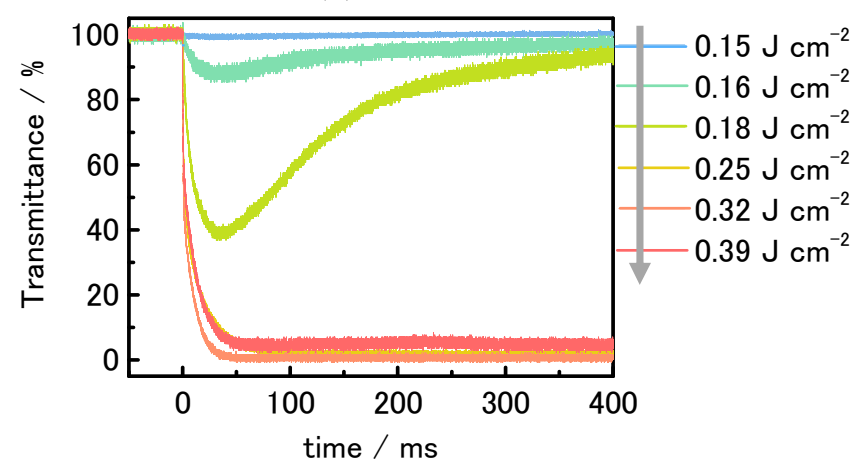

Figure 2. Representative time profiles of the optical transmittance in aqueous (a) $r-54$ and (b) $r-68$ solutions $(5.0 \mathrm{wt} \%)$ in the presence of Crystal Violet $(0.50 \mathrm{mM})$ for various incident heating laser pulse energies. The base temperature (temperature before T-jump) was $31.8^{\circ} \mathrm{C}$ for $r-54$ and $33.8^{\circ} \mathrm{C}$ for $r-68$. 
(a) $r-54$

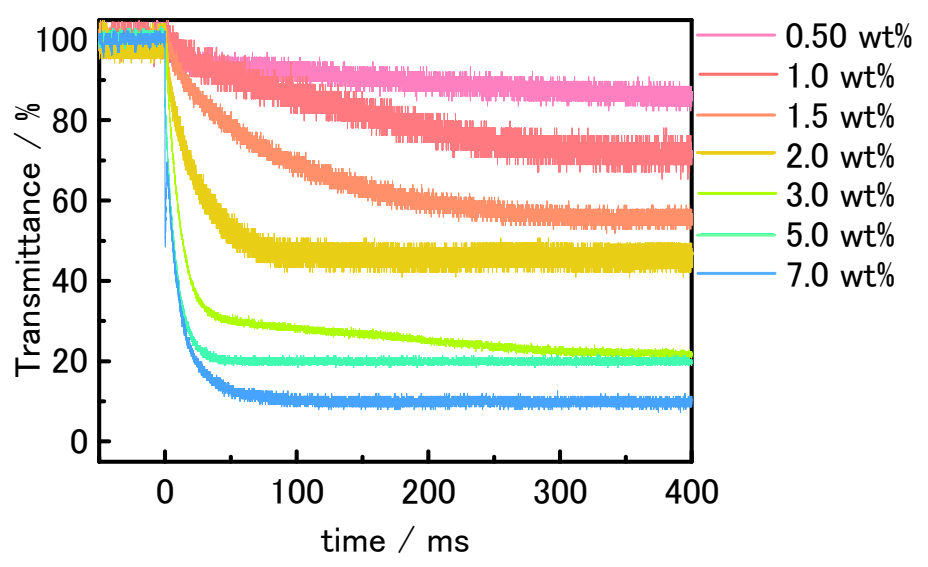

(b) $r-68$

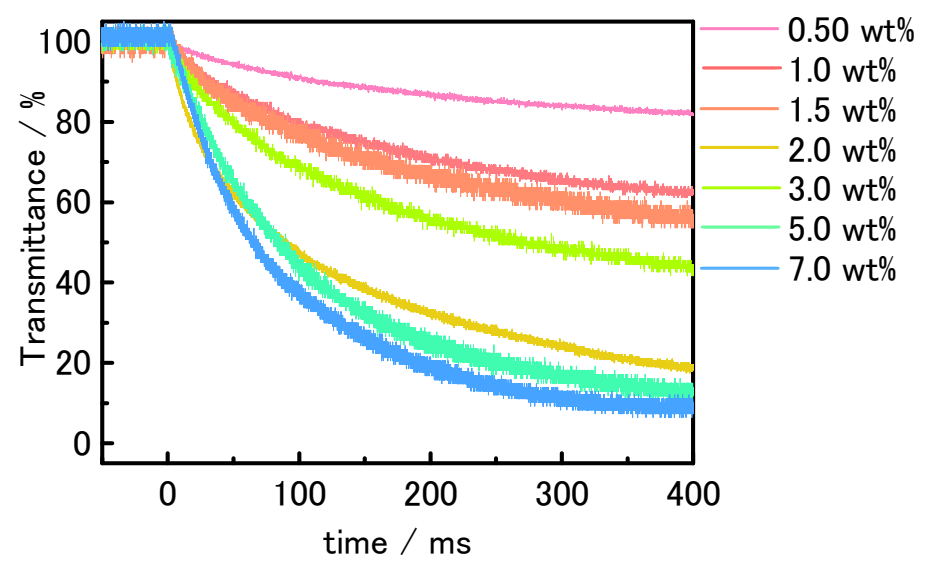

Figure 3. Time profiles of the optical transmittance for various concentrations of (a) $r-54$ and (b) $r-68$ in aqueous solutions $(0.50-7.0 \mathrm{wt} \%)$ in the presence of Crystal Violet $(0.50 \mathrm{mM})$. The base temperature (temperature before T-jump) was $31.8{ }^{\circ} \mathrm{C}$ for $r-54$ and $33.8^{\circ} \mathrm{C}$ for $r-68$. 
(a) $r-54$ and $r-68$

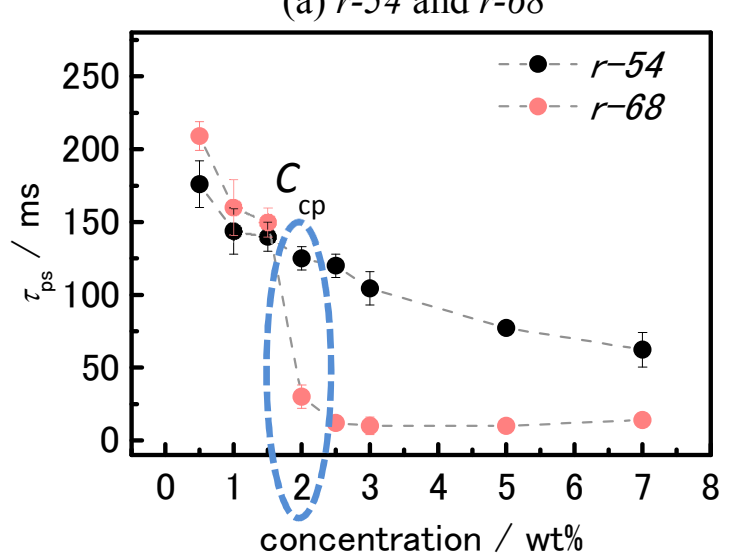

(b) $r-66$

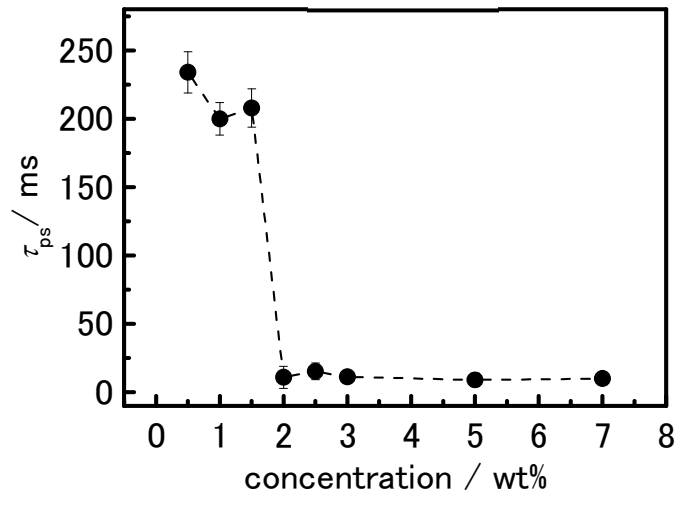

(c) $r-67$

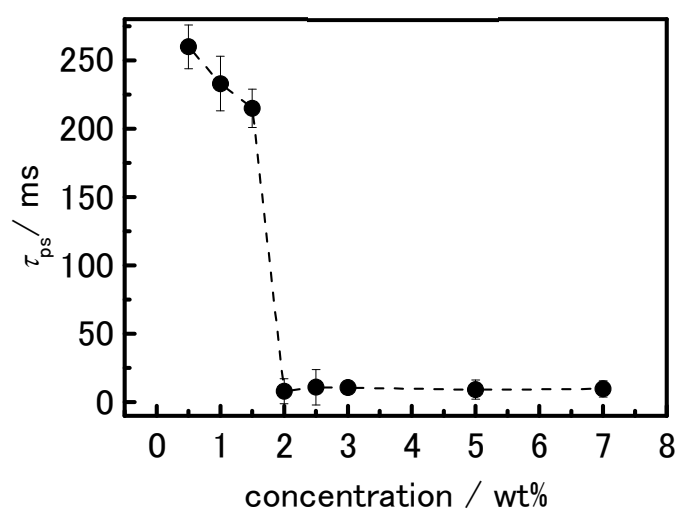

Figure 4. Phase separation time constant as a function of concentration ranging from $0.50 \mathrm{wt} \%$ to $7.0 \mathrm{wt} \%$ for (a) $r$-54 (black plots) and $r$-68 (red plots), (b) $r$ - 66 , and (c) $r-67$ in the presence of Crystal Violet $(0.50 \mathrm{mM})$. 


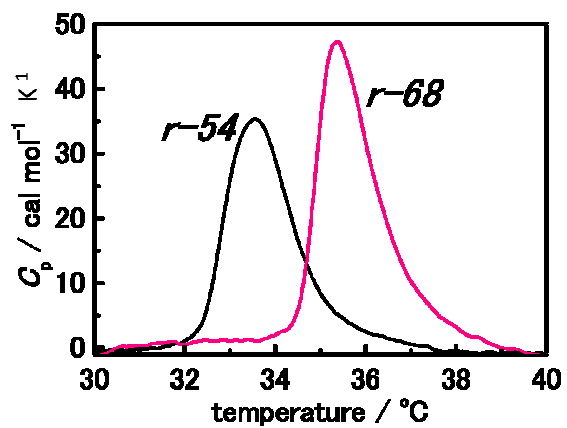

Figure 5. Heat capacity changes during phase transition/separation of aqueous $r$-54 (black) and $r$-68 (red) solutions $(1.0 \mathrm{wt} \%)$. Heating rate $=$ $1.0 \mathrm{~K} \mathrm{~min}^{-1}$ 
Table 2. Thermodynamic parameters during phase transition/separation $\left(30-40{ }^{\circ} \mathrm{C}\right)$ of aqueous $r$-54 and $r$-68 solutions (1.0wt\%). Experimental errors in these values are less than $2.0 \%$.

\begin{tabular}{cccccc}
\hline Sample name & $T_{\mathrm{p}} /{ }^{\mathrm{o}} \mathrm{C}^{(1)}$ & $\Delta T_{1 / 2} /{ }^{\mathrm{o}} \mathrm{C}^{(2)}$ & $\Delta H_{\mathrm{c}} / \mathrm{J} \mathrm{mol}^{-1(3)}$ & $\Delta H_{\mathrm{v}} / \mathrm{kJ} \mathrm{mol}^{-1(4)}$ & $n_{\text {coop }}{ }^{(5)}$ \\
\hline$r-54$ & 33.6 & 1.68 & 288 & 22.3 & 77 \\
$r-68$ & 35.4 & 1.42 & 332 & 29.3 & 88 \\
\hline
\end{tabular}

(1) Temperature of the endothermic peak. ${ }^{(2)}$ Full-width at half maximum of the endothermic peak. ${ }^{(3)}$ calorimetric enthalpy. ${ }^{(4)}$ van't Hoff enthalpy calculated by the equation $\Delta H_{\mathrm{v}}=4 R T_{\mathrm{p}}{ }^{2} / \Delta T_{1 / 2}$ (R: gas constant) ${ }^{(5)}$ Number of cooperative units, $n_{\text {coop }}=$ $\Delta H_{\mathrm{v}} / \Delta H_{\mathrm{c}}$. 
(a) $r-54$

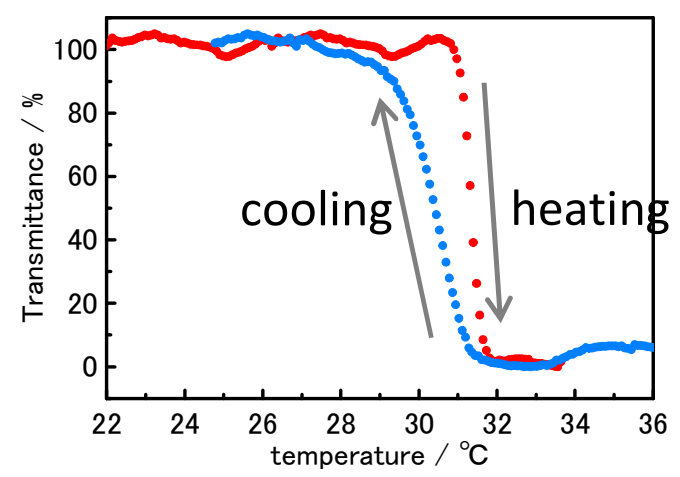

(b) $r-68$

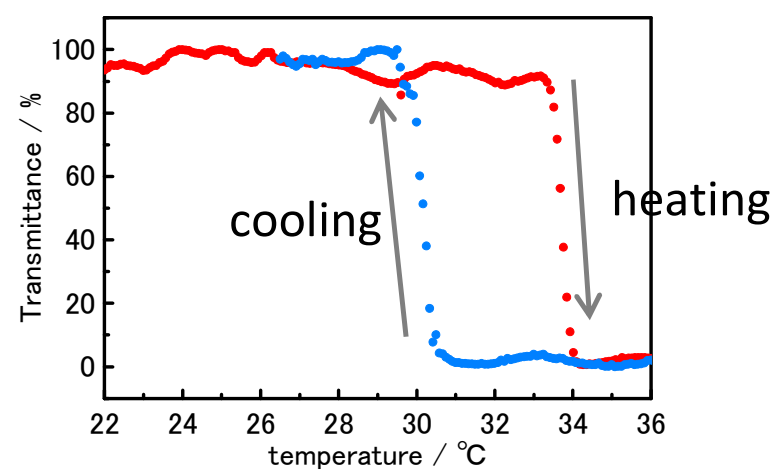

Figure 6. Temperature dependences of the optical transmittance at $532 \mathrm{~nm}$ for aqueous (a) $r$-54, (b) $r-68$ solutions. The red and blue curves show the transmittance changes upon heating and cooling, respectively. Heating and cooling rate $=0.2{ }^{\circ} \mathrm{C} \mathrm{min}^{-1}$. 


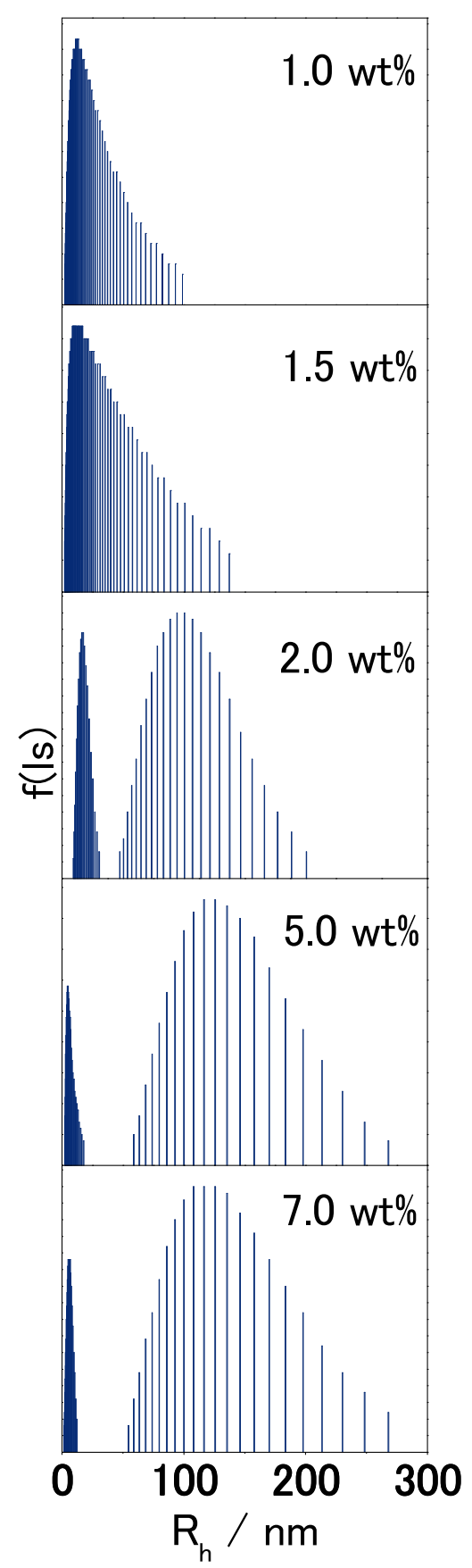

Figure 7. Representative results for the concentration dependence of the $R_{\mathrm{h}}$ distribution for $r-68$. The concentration of the sample solution is given in the figure (1.0 $-7.0 \mathrm{wt} \%)$. 


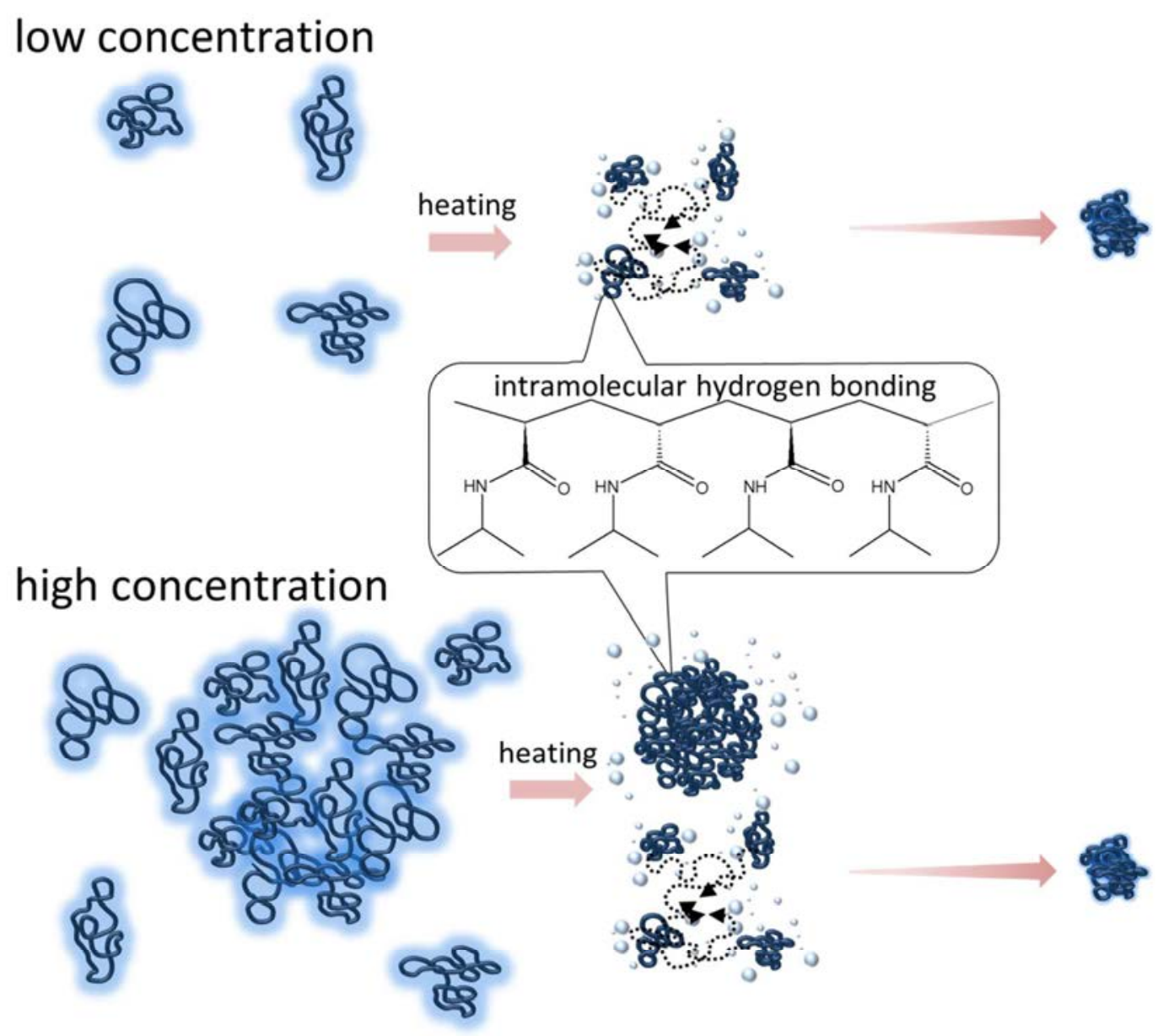

Figure 8. Schematic illustration of the phase separation dynamics of an aqueous syndiotactic-rich PNIPAM solution at various concentrations. 


\section{TOC Graphics}

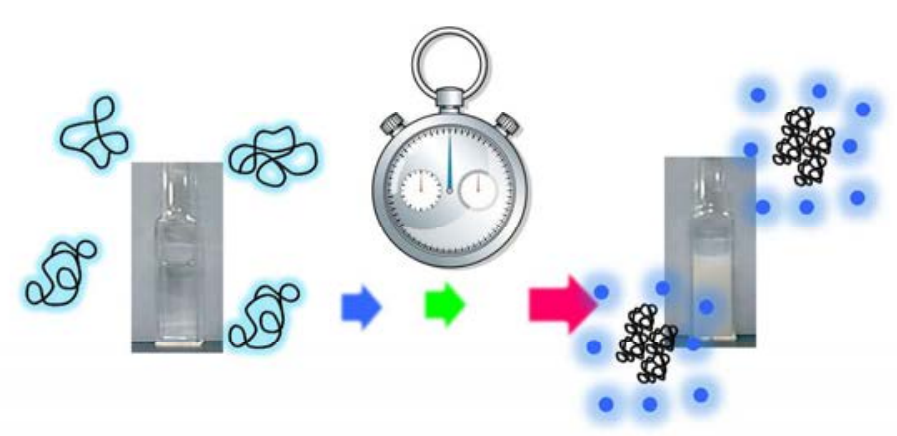

